\title{
An Ultrahigh Precision Network Time Synchronization Technology
}

\author{
Yu Quan, Liangfu Peng*, Yongqing He \\ College of Electrical \& Information Engineering, Southwest Minzu University, Chengdu, China \\ Email: 467821361@qq.com
}

\begin{abstract}
For clock synchronization, how to realize sub-nanosecond time synchronization in Ethernet is a technical problem. White Rabbit (WR) time synchronization is a new time synchronization technology developed on the basis of Precision Time Protocol (PTP). WR is based on Gigabit Ethernet, using physical layer clock distribution technology to achieve clock frequency distribution; using PTP to achieve timestamp synchronization; using digital dual mixer time difference (DDMTD) phase detector to improve the synchronization precision of timestamp to subnanoseconds. This paper introduces the WR network hierarchical topology; focuses on the special link delay mode of WR and the specific process and steps of the synchronization implementation, and deduces the link delay and offset; analyzes the key techniques of WR time synchronization technology; finally describe the application and development prospects of this technology at home and abroad. WR can improve the accuracy of time synchronization to the sub-nanosecond level, which can be widely used in important fields such as communication, navigation, electricity, industry and national defense construction.
\end{abstract}

Keywords: White Rabbit, Precision Time Protocol, Digital Dual Mixer Time Difference, Ethernet.

\section{Introduction}

In modern communication and industrial network, the normal operation of most services requires the system to provide the uniform time signal, namely clock synchronization, for each equipment module to meet the precision requirements. With the wide application of Ethernet technology in many fields, the real-time requirement of Ethernet data transmission is more and more strict. In terms of both precision and low cost, existing time synchronization technologies such as NTP/SNTP and PTP have no practical advantages in real-time Ethernet. Problems such as low synchronization accuracy (microsecond scale) and increased error rate make them increasingly unable to meet the requirements of higher time synchronization accuracy between devices [1]. In order to realize sub-nanosecond clock synchronization in distributed network system and ensure the certainty of time delay in data transmission. Based on the PTP clock synchronization method, European Organization for Nuclear Research (CERN) and Gesellschaft für Schwerionenforschung (GSI) have proposed a time synchronization technology, White Rabbit (WR), which can meet the requirements of ultra-precise measurement and control. For the defect of poor PTP timestamp accuracy, WR proposes to improve the accuracy by phase discrimination $[2-3]$.

Firstly, this paper introduces the hierarchical topology of WR network, derives the formula of link delay based on WR link delay mode, and obtains the relevant parameters of calculation delay, considers and corrects the uplink and downlink asymmetry in the allocation of round-trip delay, and obtains the relative delay coefficient of the link asymmetry. Secondly, the synchronization process of WR is studied, and the establishment stage of WR link is analyzed mainly on the basis of PTP synchronization link. Then, the key technologies of WR are studied, by using the phase detector to accurately measure the phase difference between the recovered clock and the reference clock, and correct the time stamp, the measurement accuracy of the time stamp is improved. Compared with previous synchronization technologies, WR time synchronization technology has higher accuracy and better comprehensive performance. Finally, the application of WR time synchronization technology in related fields at home and abroad is introduced, and the future application and research of this technology in many industries are prospected. 


\section{Basic Principles of WR}

With the increasing application of measurement and control technology in distributed systems, the previous time synchronization technology has been difficult to meet the needs of high-precision synchronization. Although WR was originally designed for accelerator control, its great advantages of long distances, multiple nodes and high accuracy make it widely used in large-scale scientific experiments and many technical fields. Based on Synchronous Ethernet, WR uses the existing Ethernet data line to transmit, and the clock link is multiplexed with the data link, which makes the originally rare bandwidth resources more fully utilized. Therefore, WR can simplify the structural design of the large-scale distributed network ground-based timing system, improve the synchronization performance, and improve the navigation positioning timing accuracy. It is a high-reliability, low-cost synchronization scheme with important theoretical significance and practicality [4].

\subsection{The Origin of WR}

WR time synchronization technology is a distributed system synchronization scheme based on Synchronous Ethernet, PTP protocol and digital dual mixer time difference technology. It realizes subnanosecond time synchronization between nodes in the packet network. The protocol results from the combination of PTP with two further requirements: precise knowledge of the link delay and clock syntonization over the physical layer with Synchronous Ethernet. In WR, accurate synchronization of link is accomplished by computing hardware timestamps and link asymmetry coefficients. PTP has improved the precision of time synchronization to the sub-microsecond level, but it is difficult to achieve more accurate time synchronization due to its own technical defects. Its limitations are mainly reflected in the following three aspects [5-7]:

(1) PTP assumes that the uplink and downlink between the master and slave nodes are completely symmetrical. After obtaining the round-trip delay between the master and slave nodes, it directly distributes them equally, without considering the asymmetry of transmission link as a factor affecting the synchronization accuracy.

(2) All the clocks in the standard Ethernet node is running independently, will surely there is a certain frequency deviation between the master and slave nodes, and the deviation will be with the accumulation of running time, thus to compensate the deviation must be real-time, this requires synchronization between the master and slave nodes packet switching frequency is high enough, the link to bring very great load.

(3) The timestamp is used to calculate the link delay and deviation between the master and slave nodes. The size of the timestamp resolution determines the accuracy of the time synchronization. However, the resolution of the timestamp in PTP is limited by the clock frequency and cannot provide a high resolution timestamp. For example, in Gigabit Ethernet with a clock frequency of $125 \mathrm{MHz}$, the time resolution is only $8 \mathrm{~ns}$.

In order to overcome the above limitations, WR time synchronization technology establishes a special link delay model, introduces a relative delay coefficient, uses Synchronous Ethernet to achieve frequency distribution. And uses the phase-detection technology to improve the accuracy of the time stamp in the master-slave node, finally improves the precision of synchronization to sub-nanosecond.

\subsection{WR Network Topology}

A single-link WR link is formed by a pair of nodes. The master node uses a traceable clock to encode data over the physical layer, while the slave recovers this clock (synchronization) and bases its timekeeping on it. Absolute time synchronization between master and slave is achieved by adjusting the clock phase and offset of the slave to that of the master. The phase and offset adjustment is done through the two-way exchange of PTP sync messages, which are time-stamped to achieve subnanosecond accuracy.

Multi-link WR networks are obtained by chaining WR links forming a hierarchical topology, see Fig. 1. In a WR network, some applications need WR and PTP nodes to coexist, PTP type devices can work normally in the WR network as well. As a result of this topology, a WR network consists of two kinds of WR network devices: WR boundary clocks (WR BC) and WR ordinary clocks (WR OC). WR 
boundary clock distributes the frequency and time retrieved from the upstream link to all the downstream links, like WR switch. WR ordinary clock like WR Node [8]. A frequency traceable to a common grandmaster must be distributed over the physical layer, resulting in a cascade of master and slave nodes.

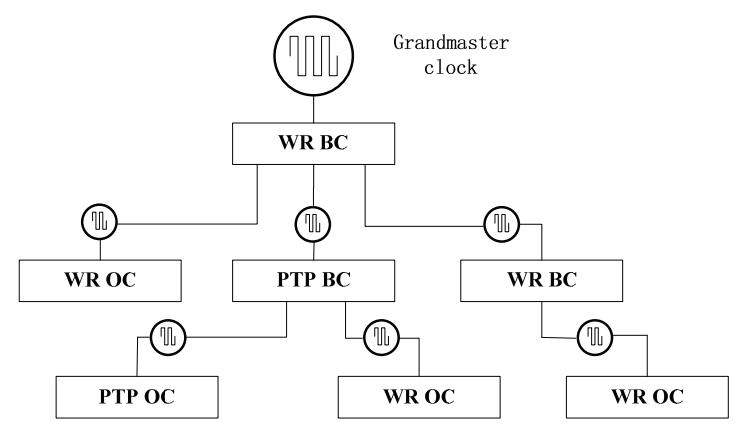

Figure 1. WR network hierarchical topology

\subsection{Link Delay Model}

WR determines and establishes a unique link delay model and defines the fixed delays caused by the transmission and reception circuits over time, as well as the variable delays that are generated in the transmission medium. The link delay model between devices is shown in Fig. 2.

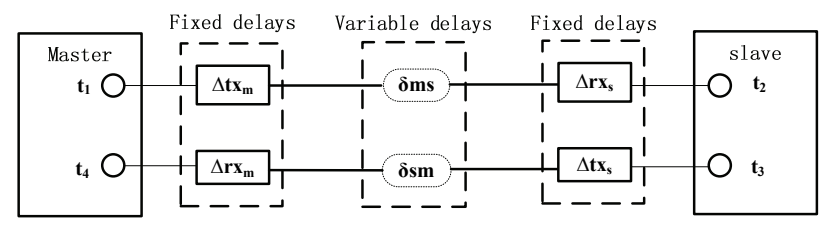

Figure 2. Delay model of a WR link

The delay of messages traveling from master to slave can be expressed as follow, due to the delay model:

$$
\begin{aligned}
& \text { delay }_{\mathrm{ms}}=\Delta_{t x_{m}}+\delta_{m s}+\Delta_{r x_{s}} \\
& \text { delay }_{\mathrm{sm}}=\Delta_{t x_{s}}+\delta_{s m}+\Delta_{r x_{m}}
\end{aligned}
$$

where $\Delta_{t x_{m}}$ and $\Delta_{t x_{s}}$ are the fixed delay due to the master's and slave's transmission circuitry; $\delta_{m s}$ and $\delta_{s m}$ are the variable delay incurred in the transmission medium; $\Delta_{r x_{s}}$ and $\Delta_{r x_{m}}$ are the fixed delay due to the slave's and master's reception circuitry.

An accurate relation between both variable delays on the transmission line is essential for obtaining an acceptable estimate of the delay asymmetry on a WR link. The relation between $\delta_{m s}$ and $\delta_{s m}$ is represented in WR by the relative delay coefficient $(\alpha)[9]$. When a single-mode fiber is used as bidirectional communication medium, it can be shown that both variable delays are related by an equation of the form (3). Thus can consider the asymmetry of the transmission link as an important factor affecting the synchronization accuracy by the link delay model.

$$
\delta_{m s}=(1+\alpha) \delta_{s m}
$$

\subsection{Synchronization Process}

WR is a time synchronization technology that is improved on the basis of PTP and achieves subnanosecond ultra-high precision. WR uses the link delay model to obtain an accurate delay estimation. WR introduces the White Rabbit Link Setup (WR Link Setup), which is a process for establishing the 
WR link. It includes syntonization of the local clock over the physical layer, measurement of fixed delays (calibration) and distribution of the information about fixed delays over the link.

Additional communication between the master and slave nodes needs to be done by extending the interaction of the PTP messages. WR benefits from the PTP messaging mechanism, defines an extended WR Type_Length_Value (WR TLV) to exchange WR specific messages between master and slave nodes, and uses a two-step clock delay request response mechanism to complete the synchronization. Before the synchronization of the time-stamped synchronization information, the corresponding WR node is found by sending a WR Announce message with a custom suffix. Then slave node establishes its location in the network hierarchy, the master node requests synchronization, the master and slave nodes calibrate their own fixed transmission and reception delays respectively, etc, complete the WR Link Setup process [10]. The next step is to perform synchronous message transmission according to the standard PTP delay request response mechanism, and finally achieve the purpose of master-slave node synchronization. During the completion of the WR Link Setup, communication between the WR master node and the WR slave node is performed using signaling messages carrying the WR TLV. The process of synchronizing master and slave nodes in WRPTP is shown in Fig. 3.

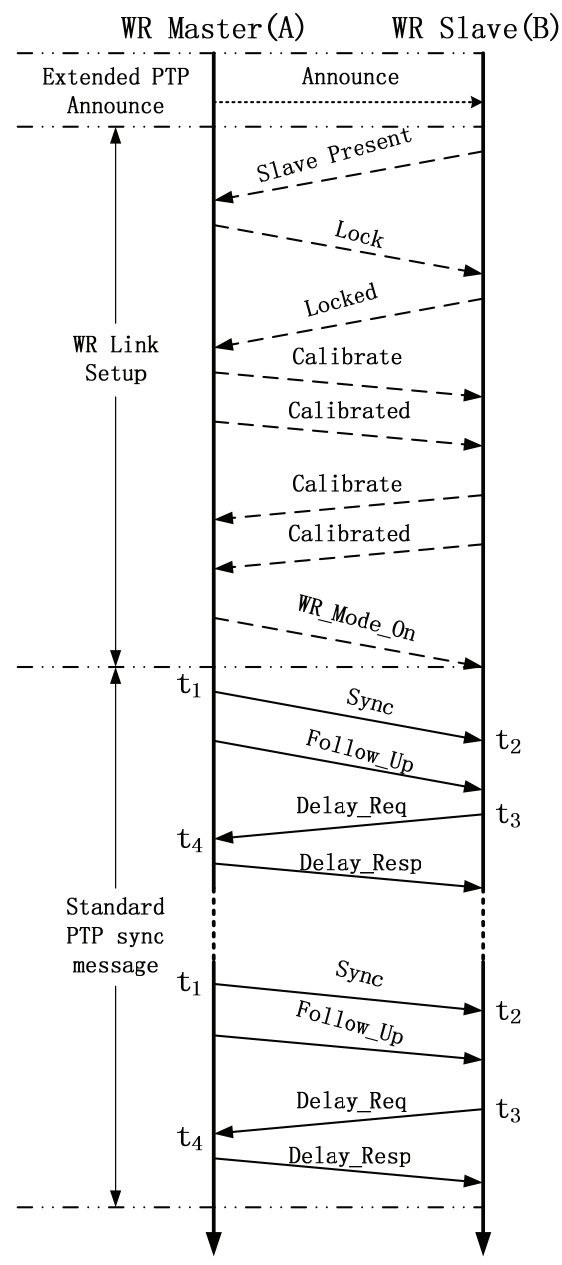

Figure 3. Simplified overview of the message flow in WRPTP

1) WR Node A which is in PTP MASTER state periodically sends WR Announce messages with a custom suffix.

2) WR Node B receives Announce message(s), recognizes the WR Announce message and uses the modified BMC algorithm to establish its place in the WR network hierarchy.

3) WR Node B enters WR Slave mode and starts the WR Link Setup by sending the 
SLAVE_PRESENT WR Signaling message.

4) WR Node A enters WR Master mode and sends the LOCK WR Signaling message to request the WR Slave to start syntonization.

5) The WR Slave sends the LOCKED WR Signaling message as soon as the syntonization process is finished.

6) The WR Master sends the CALIBRATE WR Signaling message to request the calibration pattern. It calibrates its transmission and reception fixed delay.

7) The WR Master sends the CALIBRATED WR Signaling message as soon as the calibration is finished.

8) The WR Slave sends the CALIBRATE WR Signaling message to request the calibration pattern. It calibrates its transmission and reception fixed delay.

9) The WR Slave sends the CALIBRATED WR Signaling message as soon as the calibration is finished.

10)The WR Master sends the WR_MODE_ON WR Signaling message to indicate completion of the WR Link Setup process.

11)The WR Master periodically sends a Sync message (timestamped on transmission, $t_{1}$ ) followed by a Follow_Up message which carries $t_{1}$.

12)The WR Slave receives the Sync message sent by the master (timestamped on reception, $t_{2}$ ).

13)The WR Slave receives the Follow_Up message sent by the master.

14)The WR Slave sends a Delay_Req message (timestamped on transmission, $t_{3}$ ).

15)The WR Master receives the Delay_Req message sent by the slave (timestamped on reception, $t_{4}$ ).

16)The WR Master sends a Delay_Resp message which carries $t_{4}$.

17) The WR Slave receives the Delay_Resp.

18)The WR Slave adjusts its clock using the clock offset and the link delay calculated with the timestamps $\left(t_{1}, t_{2}, t_{3}, t_{4}\right)$. This results in the slave's synchronization with the master clock with subns accuracy.

19) Repeat 1, 11-18.

Through the analysis of the WR time synchronization process, utilize the standard PTP synchronization mechanism and the four timestamps $\left(t_{1}, t_{2}, t_{3}, t_{4}\right)$ obtained during its synchronization, thus the mean path delay is defined as:

$$
\mu=\frac{\left(t_{2}-t_{1}\right)+\left(t_{4}-t_{3}\right)}{2}
$$

What is more, the transmission delays $t_{2}-t_{1}$ and $t_{4}-t_{3}$ can be expressed in terms of WR's delay model:

$$
\begin{aligned}
& t_{2}-t_{1}=\Delta_{t x_{m}}+\delta_{m s}+\Delta_{r x_{s}}+\text { offset } t_{m s} \\
& t_{4}-t_{3}=\Delta_{t x_{s}}+\delta_{s m}+\Delta_{r x_{m}}-\text { offset }{ }_{m s}
\end{aligned}
$$

where offset $t_{m s}$ is the time offset between the slave's clock and the master's. Combining the equations of (4), (5), (6) above we obtain:

$$
2 \mu=\Delta+\delta_{s m}+\delta_{m s}
$$

Equation (7) also shows the round-trip delay of the link delaymm, which accounts for all fixed delays in the link, i.e.:

$$
\Delta=\Delta_{t x_{m}}+\Delta_{r x_{s}}+\Delta_{t x_{s}}+\Delta_{r x_{m}}
$$

Using equations (3) and (7), two variable delays between the master and slave nodes can be obtained:

$$
\begin{gathered}
\delta_{m s}=\frac{1+\alpha}{2+\alpha}(2 \mu-\Delta) \\
\delta_{s m}=\frac{2 \mu-\Delta}{2+\alpha}
\end{gathered}
$$

\section{Key Technologies of WR}

\subsection{Synchronous Ethernet}

Synchronous Ethernet technology is a key technology for synchronizing the frequency of the master and slave clocks before the PTP synchronizes. Responsible for the distribution time and frequency signals of 
physical layer in the WR network; obtaining a fixed link delay and transmitting the delay information to the upper layer; provide sufficiently accurate timestamp. In standard Ethernet, the clock of each node is dependent on the local crystal oscillator inside the node, so the clock signal of each node will definitely be different. In Synchronous Ethernet, all nodes form a network clock topology, the reference clock encodes the output data stream, and the same clock signal can be detected at the other end of the physical link [11]. The detected clock signal can be further sent and will go back to the sender after a loop. After the slave node passes through the phase-locked loop inside the node to eliminate jitter, the clock of the master node is recovered from the data link by using the clock recovery technology, so that all slave nodes in the network can keep accurate synchronization of clock frequency with the master node [12].

In Synchronous Ethernet, since each node has the same clock signal, phase detection techniques can be used to measure the delay. The phase measurement can improve the accuracy of the time stamp, obtain the value of the fixed delay and distribute it over the physical layer, and then calculate the value of the link asymmetry coefficient. Ethernet is a fairly mature mainstream data transmission technology, and many network device interfaces can be used universally. Therefore, the use of mature synchronous Ethernet technology to complete the transmission of clock signals on low-cost transmission media (such as fiber and cable) is a major advantage of WR technology.

\subsection{Precise Time Protocol (PTP)}

With the increasing application of measurement and control technology in distributed systems, previous time synchronization protocols have been difficult to meet their performance requirements. PTP time synchronization protocol capable of achieving sub-microsecond synchronization accuracy came into being. PTP is a packet-based protocol designed to synchronize devices in distributed systems [13]. The standard defines two kinds of messages which are exchanged between PTP nodes: event messages and general messages. Both the time of transmission and reception of event messages are need to recorded. General messages are used by PTP nodes to identify other PTP nodes, establish clock hierarchy and exchange data. Fig. 4 presents the messages used when the delay request-response mechanism (with a two-step clock) is used, which is the case in White Rabbit. It can be said that PTP is the core technology that WR time synchronization technology can achieve.

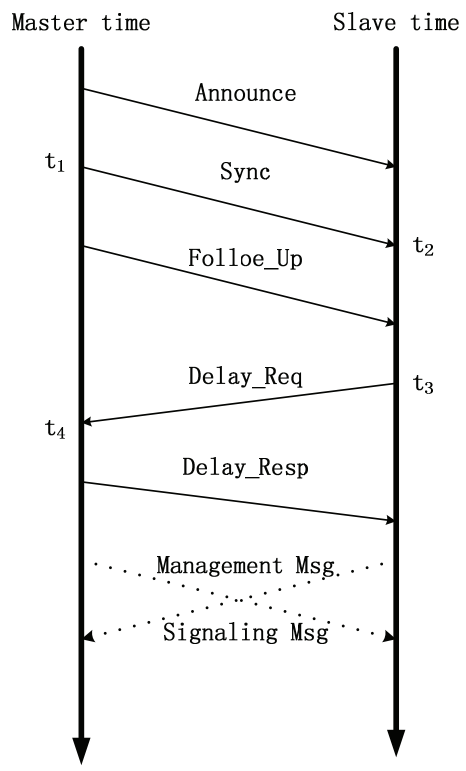

Figure 4. PTP messages used by WRPTP

Sync Messages and Delay_Req Messages are time stamped $\left(t_{1}, t_{2}, t_{3}, t_{4}\right)$ and these timestamps are used to calculate the offset and the link delay between the nodes exchanging the messages. Follow_Up 
Messages and Delay_Resp Messages are used to send timestamps between master and slave. Management Messages are used for configuration and administrative purposes. Signaling Messages are used for communication between clocks [14]. In the PTP delay request response mechanism, the transmission and reception process of the message is exactly the same as the standard PTP synchronization process (ie, steps 11-18) in the synchronization process of the WR master-slave node, the description will not be repeated here.

\subsection{Digital Dual Mixer Time Difference (DDMTD)}

Digital dual mixer time difference(DDMTD) is one of the key technologies for WR protocol to achieve sub-nanosecond synchronization accuracy. WR requires precise timestamps those shall be sufficient to take advantage of the calculated asymmetry, in particular the precision of the reception timestamps $\left(t_{2}\right.$, $\left.t_{4}\right)$ needs to be enhanced. And brings time synchronization technology to the sub-nanosecond order. This can be done by using the common notion of frequency distributed with SyncE to cast the problem of timestamping into a phase detection measurement. The traditional phase detector realizes the corresponding function by analog method, and the resolution and linearity have reached a high level. But all of them are based on the addition of additional devices such as mixers and filters, this will not only increase costs but also increase the difficulty of design for WR multiport device applications [15-17]. In WR, the advantages of double mixing time difference method such as flexible sampling time and direct measurement of phase difference are used to digitize it to realize the digital double mixing phase discriminator. On the basis of PTP time synchronization technology, the method of adding digital double mixing phase discriminator for phase measurement is adopted to improve the measurement accuracy of time stamp. Compared with the traditional mixer, DDMTD can achieve sub-picosecond precision phase without using modules such as charge pump, and has the characteristics of no dead zone and full-scale linearity. The structure of the digital dual mixer time difference system is shown as in Fig. 5 .

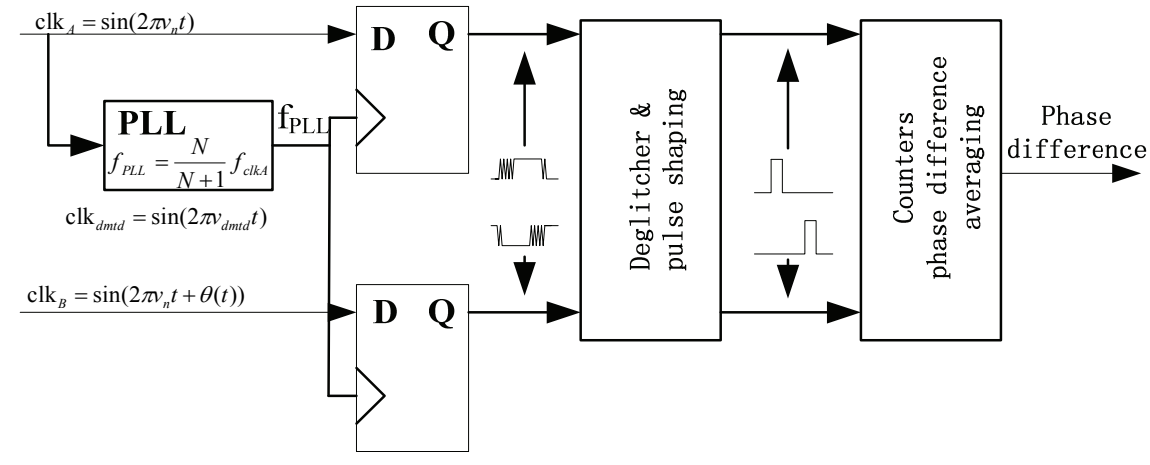

Figure 5. Structure of DDMTD

The goal of the DDMTD consists in converting a phase shift from the high frequency domain into the low frequency domain, thus providing a very large time difference multi-plication effect. This technique has the benefit of cancelling most of the common clock phase noise. The DDMTD mixer replaced by a simple D flip-flop, and the reference clock is generated by an input clock via a phase-locked loop (PLL), the frequency of the reference clock has a slight difference (a few $\mathrm{Hz}$ ) from the frequency of the input signal. The input signals $\left(\mathrm{clk}_{A}, \mathrm{clk}_{B}\right)$ are respectively mixed with a reference source $\left(\mathrm{clk}_{d m t d}\right)$ to obtain a beat frequency signal, then two beat signals enter the time interval counter for measurement and counting. The relationship between the input signal and the reference signal is as shown in equation (11), the role of the reference clock is to magnify the phase difference between the two input signals by a factor of $N$ to make it easier and more accurate to measure.

$$
v_{d m t d}=\frac{n}{n+1} v_{n}
$$

After two input signals with very close frequencies are processed by the D flip-flop, and a low frequency signal is obtained at the output. By measuring the phase difference of the output signal of the 
D flip-flop, the original phase difference of the input signal can be obtained according to the formula, thereby achieving the purpose of synchronization. The symmetrical structure and the same reference clock based on the double mixing time difference principle make the noise impact of the two beat signals almost the same, which can greatly offset the system noise [18-19].

The timestamping unit produces timestamps on both the rising and falling edges of the clock. WR uses the clock loopback technique to provided with the phase measurement of the round-trip phase ( phase $_{\mathrm{mm}}$ ) in case of the master, the setpoint phase $\left(\right.$ phase $_{\mathrm{s}}$ ) in case of the slave and sent to the phase adjustment module for phase adjustment and synchronization. The phase adjustment module is composed of a phase detector, a filter circuit and a voltage controlled oscillator, and constitutes a phase locked loop circuit to realize corresponding functions [20]. The phase adjustment of the WR master to slave node is shown in Fig. 6.

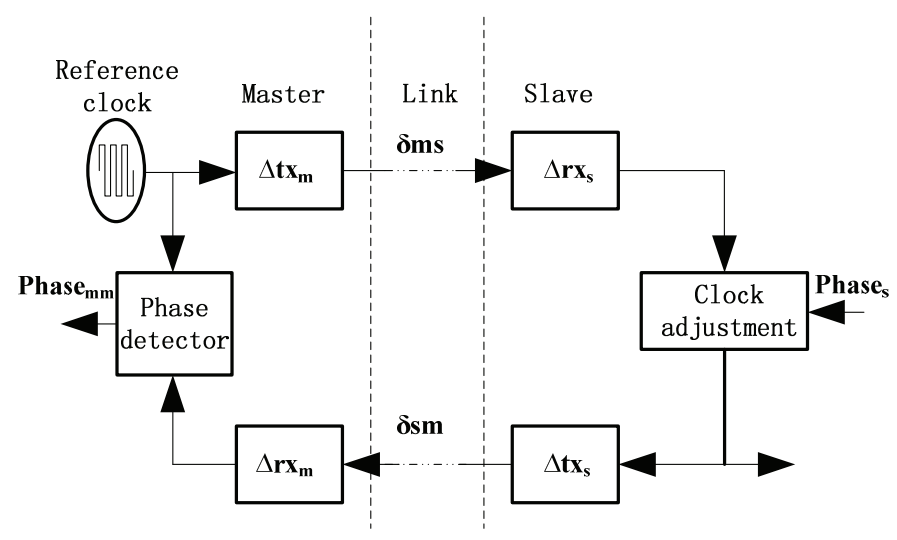

Figure 6. The phase adjustment of the WR master to slave node

The value of phase $\mathrm{mm}_{\mathrm{m}}$ can be obtained in the master and expressed as equation(12):

$$
\text { phase }_{\mathrm{mm}}=\frac{\Delta+\delta_{m s}+\delta_{s m}}{T_{r e f}}
$$

where $T_{\text {ref }}$ is the period of reference clock. The accuracy of timestamps $t_{2}$ and $t_{4}$ is enhanced by using the knowledge of the round-trip phase phase ${ }_{\mathrm{mm}}$ and the slave setpoint phase $\mathrm{s}_{\mathrm{s}}$. And we can calculate the precise round-trip delay delaym $y_{m m}$ of the link.

$$
\text { delay }_{m m}=\left(t_{4 p}-t_{1}\right)-\left(\mathrm{t}_{3}-t_{2 p}\right)
$$

Assuming that the link delay stays constant, increasing a certain amount of phase $\mathrm{s}_{\mathrm{s}}$ will cause $t_{4 p}$ to also increase by the same amount, while $t_{2 p}$ correspondingly reduces the same amount. It can be seen

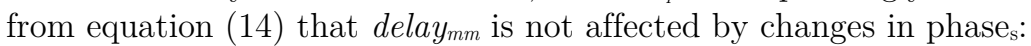

$$
\text { delay }_{m m}=\left(t_{4 p}-t_{1}\right)-\left(t_{3}-t_{2 p}\right)=t_{4 p}+\text { phase }-t_{1}-t_{3}+t_{2 p}-\text { phase }
$$

Having obtained the values of round-trip delay delaym and link asymmetry $(\alpha)$, we can calculate the one-way master to slave delay, delayms, and the offset of master to slave, offset $t_{m s}$. WR slave node can adjust the phase of the clock to improve the accuracy of the synchronization. The corresponding calculation process is as follows:

$$
\begin{gathered}
\text { delay }_{\mathrm{mm}}=\Delta+\delta_{\mathrm{ms}}+\delta_{S m} \\
\delta_{m s}=\frac{1+\alpha}{2+\alpha}\left(\text { delay }_{m m}-\Delta\right) \\
\text { delay }_{\mathrm{ms}}=\frac{1+\alpha}{2+\alpha}\left(\text { delay }_{m m}-\Delta\right)+\Delta_{t x_{m}}+\Delta_{r x_{s}} \\
\text { offset }_{m s}=t_{1}-t_{2 p}-\text { delay }_{m s}
\end{gathered}
$$




\section{Application and Prospects}

Many large-scale scientific research experiments at home and abroad need to achieve ultra-high-precision time synchronization in a large-area space environment. The WR technology capable of providing subnanosecond synchronization has been widely applied. WR time synchronization technology appears in the most famous applications in China at Large High Altitude Air Shower Observatory (LHAASO). The LHAASO in Daocheng is the cosmic ray detector with the highest altitude and the largest distribution area in the world. The purpose is to observe cosmic rays, study the origin, acceleration and propagation mechanisms of high-energy cosmic rays, and explore new physical phenomena in the universe. The LHAASO has four types of large detectors, each of which requires ultra-precise time synchronization technology to ensure proper operation of the equipment. For example, the Water Cherenkov Detector Array (WCDA) as its core detector has the advantages of wide distribution area and a large number of detector channels. Front-end electronics adopts a distributed digital scheme, which uses WR time synchronization technology to distribute clock signals to each node to achieve ultra-high-precision synchronization of each slave node and master node [21-22].

In foreign countries, CERN Neutrinos to Gran Sasso (CNGS) neutrino experiment is used to study neutrino oscillations in Europe. Due to the need to send the resulting $\mu$ neutrino beam to the Gran Sasso National Laboratory (LNGS) in Italian, more than 700 kilometers away. LNGS adopts WR ultrahigh-precision time synchronization technology, which makes full use of its ultra-high-precision synchronization of sub-nanoseconds when transmitting over long distances. In addition, the literature [23] pointed out the Russian Tunka-HiS-CORE cosmic ray detector, KM3NeT which under the Mediterranean and Cherenkov Telescope Array (CTA) also uses WR as time synchronization solution. The French National Metrology Laboratory applied WR time synchronization technology to the interior of the Paris Observatory to spread UTC time.

With the improvement of the degree of informatization in various industries, time synchronization has penetrated into all walks of life in the national economy. Therefore, higher requirements and more urgent expectations have been put forward for the development of time synchronization technology. WR time synchronization technology can be applied not only in large-scale physics experiments, but also widely used in telecom operations, electricity, national defense and transportation. The upcoming $5 \mathrm{G}$ era will truly realize the interconnection of all things, with low latency, wide coverage, and more secure requirements. To meet the critical needs of low latency, ultra-high precision time synchronization technology will play an important role in technology development. The demand for time synchronization in the electricity industry is also very urgent and the accuracy required is very high. There are a large number of unattended substations in the electricity system. And there are many monitoring devices in the substation to assist in electricity production. The coordinated operation of these devices ensures the normal operation of the power system. In these devices, high-precision time information must be provided to accurately record the time of the event and the fault, and provide scientific clues and basis for the operation and maintenance of the system. In recent years, the deepening of national defense modernization construction, the demand for time synchronization accuracy in the field of defense communications has also become increasingly prominent. As more and more high-tech weapons and equipments are used in the military, the requirements for time synchronization are correspondingly higher and higher, and the time synchronization accuracy of some precision-guided weapons is within the order of nanoseconds. Ultra-precise time synchronization technology can provide sufficient guarantee for the victory of high-tech warfare under the conditions of informationization. Therefore, in the era of high informationization now and in the future, WR time synchronization technology will certainly have a larger and wider application and development.

\section{Conclusion}

As the scale of the industry gradually increases, the following network system presents a more decentralized trend. Network equipment in many systems has extremely strict requirements on real-time, which leads to higher and higher requirements on the accuracy of time synchronization between various node devices in the network system. WR clock synchronization technology improves the precision of clock synchronization to a certain extent. It does not need satellite signal coverage to support, and can 
be applied indoors and in places where satellite signals cannot be received. It is at a relatively high level in terms of security, reliability and controllability. As time synchronization technology plays an important role in supporting the development of national economy, science and technology, military and other aspects, the research results of WR ultra-high precision time synchronization technology have a very broad application prospect, and will become the booster to promote the continuous development of science and technology in the world.

Acknowledgements. The work of this paper is supported by the Southwest Minzu University Graduate Innovative Research Project (Master Program CX2019SZ13). A special acknowledgement should give to Southwest Minzu University for its experimental conditions and technical support.

\section{References}

1. Tomasz Wlostowski. Precise time and frequency transfer in a White Rabbit network [D]. Warsaw University of Technology, 05/2011.

2. Chen Xi, Teng Ling, Gao Qiang, et al. Error Analysis of Time Synchronization in NTP and PTP [J]. Journal of Astronautic Metrology and Measurement, 2016, 36(3): 35-40.

3. Pan Weibin. Research on the High Precision Time Measurement System for the LHAASO Project [D]. Tsinghua University, 2014.

4. Pan Weibin, Gong Guanghua, Du Qiang, et al. The White Rabbit Protocol and its Applications in Scientific Experiments $[\mathrm{C}] / /$ China Nuclear Science and Technology Progress Report (Volume III) - Proceedings of the 2013 Annual Meeting of the Chinese Nuclear Society. 2013.

5. Murakami T, Horiuchi Y. A master redundancy technique in IEEE 1588 synchronization with a link congestion estimation[C]// IEEE International Symposium on Precision Clock Synchronization for Measurement. IEEE, 2010.

6. Kaur N, Tuckey P, Pottie P E. Time transfer over a White Rabbit network $[\mathrm{C}] / /$ European Frequency \& Time Forum. IEEE, 2016.

7. PENG Chengjin, YANG Jun, ZHANG Ming, LI Shiguang. Development and Application of WR. JOURNAL OF ASTRONAUTIC METROLOGY AND MEASUREMENT, 2018, 38(5): 7-15.

8. Dierikx E, Wallin A, Fordell T, et al. White Rabbit Precision Time Protocol on Long Distance Fiber Links [J]. IEEE Transactions on Ultrasonics, Ferroelectrics, and Frequency Control, 2016:1-1.

9. Li Hongming, Gong Guanghua, Pan Weibin. Method to Measure Fiber Asymmetry in WR [C]// Proceedings of the 17th National Conference on Nuclear Electronics and Nuclear Detection Technology. 2014.

10. Lipinski M, Wlostowski T, Serrano J, et al. Performance results of the first White Rabbit installation for CNGS time transfer[C]// Precision Clock Synchronization for Measurement Control and Communication (ISPCS), 2012 International IEEE Symposium on. IEEE, 2012.

11. Wang K, Hu YH, He ZM, et al. Design and Implementation of IEEE 1588 High Precision Clock Synchronization Based on GPS Time Service[J]. Advanced Materials Research, 2012, 532-533:292-296.

12. Gong Guanghua, Li Hongming. High-Precision Time Distribution Based on Optical Etherne [J]. Navigation Positioning and Timing, 2017(6):68-74.

13. Zeng Dongcai. Analysis and improvement on the Effects of PHY for IEEE 1588 Clock Precision [D]. Northwest University 2013.

14. Zheng Yi. Analysis on High-precision Time Synchronization Technique Based on IEEE1588 [J]. Radio Engineering, 2019, 49(6):545-550.

15. Li Yuwei, Liu Ya, Li Xiaohui. Analysis of performance of TSC multi-channel measurement system [J]. Journal of Time and Frequency, 2011(1):9-15.

16. Zhang Dan, Zhao Jun. Application and Analysis of Correlation in Time and Frequency Measurement [J]. Automation \& Instrumentation, 2011, 26(10):51-53.

17. Dierkx E F, Wallin A E, Fordell T, et al. White Rabbit precision time protocol on long-distance fiber links [J]. IEEE Transactionson Ultrasonics, Ferroelectrics, and Frequency Control, 2016, 63(7): 945-952.

18. Zhao Kejia, Zhang Aimin, Xu Qinghua et al. Experimental research on digital DMTD measurement system [J]. Chinese Journal of Scientific Instrument, 2014(12):2858-2865.

19. He Huizheng. DESIGN AND SIMULATION OF DIGITAL DUAL MIXER TIME DIFFERENCE MEASUREMENT SYSTEM [D]. BeiJing University of Technology, 2014. 
20. He Huizheng, Zhu Jiangmiao. Principles analysis and simulation of digital dual mixer time difference measuring instrument [J]. Modern Electronics Technique, 2013(19): 61-63.

21. Chu Shaoping. Reseach of Clock Synchronization and Data Transmission in LHAASO WCDA Readout Electronics [D]. University of Science and Technology of China, 2017.

22. LI Cheng, LIU Shubin, SHANG Linfeng et al. LHAASO WCDA's Prototype Clock System [J]. Nuclear Electronics \& Detection Technology, 2013, 33(4):410-416.

23. Li Peiji, Li Wei, Zhu Xiangwei, Gong Hang. Overview of Network Time Synchronization Protocol. CEA, 2019, 55(3): 30-38. 